Research Article

\title{
Healthcare Waste Management Practice and Associated Factors among Private and Public Hospitals of Bahir Dar City Administration
}

\author{
Dereje Mesfin Assemu, ${ }^{1}$ Tadese Ejigu Tafere, ${ }^{2}$ Yared Mulu Gelaw, \\ and Getasew Mulat Bantie (D) $^{3}$ \\ ${ }^{1}$ Bahir Dar City Zonal Health Department, Bahir Dar City, Ethiopia \\ ${ }^{2}$ Department of Health Economics and Health Service Management, School of Public Health Bahir Dar University, \\ Bahir Dar City, Ethiopia \\ ${ }^{3}$ Department of Public Health, Alkan Health Science Business and Technology College, Bahir Dar City, Ethiopia
}

Correspondence should be addressed to Getasew Mulat Bantie; getasewmulat@gmail.com

Received 9 June 2020; Revised 26 August 2020; Accepted 10 October 2020; Published 23 October 2020

Academic Editor: Issam A. Al-Khatib

Copyright ( 12020 Dereje Mesfin Assemu et al. This is an open access article distributed under the Creative Commons Attribution License, which permits unrestricted use, distribution, and reproduction in any medium, provided the original work is properly cited.

\begin{abstract}
Background. Lack of an appropriate management practice of healthcare waste is a potential threat to the healthcare workers, patients, and nearby communities of the health institutions. Objective. The study aimed to assess the healthcare waste management practices (HCWMP) and associated factors among healthcare workers of private and public hospitals of Bahir Dar city administration, Ethiopia. Methods. A facility-based comparative cross-sectional study was conducted from January 2016 to April 2017. The systematic random sampling technique was employed to recruit 460 healthcare workers. The collected data entered into the EpiData software (version 3.1). The analysis was done by using SPSS software (version 20). Descriptive statistics were computed; logistic regression model was run. The model fitness was checked using Hosmer and Lemeshow goodness of fit $(p>0.05)$. A $p$ value of $<0.2$ at univariate analysis was included in the multivariable logistic regression analysis. Variables with a $p$ value of $<0.05$ were statistically associated with healthcare waste management practice at 95\% CI (AOR). Results. A total of 418 healthcare workers who participated in the study yielded a response of $90.9 \%$. About $65 \%$ (95\% CI: 61, 70) of the total respondents had good practice of healthcare waste management. More private hospitals, $79.2 \%$ (95\% CI: 73, 85), had good healthcare waste management practice compared to public hospitals, 53.5\% (95\% CI: 47, 60). Male healthcare workers (AOR $=6.43,95 \%$ CI: 1.82, 22.77) and having a functional healthcare waste management committee $(\mathrm{AOR}=6.47,95 \% \mathrm{CI}: 1.93,21.76)$ were significantly associated with HCWMP at private hospitals. For public hospitals, having a healthcare waste management committee (AOR $=1.80,95 \% \mathrm{CI}: 1.03,3.15)$ and a manual/guideline on HCWMP (AOR = 2. 43, 95\% CI: 1.20, 4.91) was significantly associated with HCWMP. Conclusions. This study revealed there is a great discrepancy in HCWMP between private and public hospitals. Male healthcare workers and having a functional healthcare waste management committee and a manual/guideline were the identified factors of HCWMP.
\end{abstract}

\section{Background}

Healthcare facilities are one of the significant sectors that have been showing improvement throughout the world during recent decades [1]. However, healthcare activities can lead to the generation of various types of waste that may have adverse effects on human health and on the environment [2].
Healthcare wastes are wastes produced by health service delivering facilities and laboratories [3]. These wastes are sharps, nonsharps, blood, body parts, chemicals, pharmaceuticals, medical devices, and radioactive materials [4]. Most of these are toxic, harmful, carcinogenic, and infectious materials $[5,6]$.

Healthcare wastes account for around 1-2\% of urban wastes, which is a critical public health issue as they 
jeopardize human and environmental health [7]. Even though the current medical waste management practices vary from hospital to hospital, the awkward areas are similar to all health service delivering facilities [8]. Healthcare waste management survey in 22 developing countries revealed that the proportion of healthcare facilities that used inappropriate waste disposal method varies from $18 \%$ to $64 \%$ [9].

Improper healthcare waste management practice affects the healthcare workers, janitors, patients, and hospital environment [4, 10-13]. A proper healthcare waste management in a hospital depends on awareness, availability of training, personal protective equipment, dedicated waste management team, good administration, careful planning, sound organization, underpinning legislation, adequate financing, full participation by trained staff, use of appropriate disposal technique, and national regulatory framework $[8,14-20]$.

Proper healthcare waste management practice can prevent transmission of infectious diseases, environmental pollution, unpleasant smells, multiplication of insects, rodents, and worms [6, 17, 21]. However, healthcare waste management has not received enough attention in recent decades in economically developing countries [22]. Many findings in developing countries on healthcare wastes management revealed that segregation, collection, and storage of waste in isolated area were not satisfactory [18]; furthermore, healthcare wastes originating from healthcare facility dumped either into their backyard in a simple pit or put in open garbage to bins on the roads [23-26].

In Ethiopia, nowadays, healthcare facilities are becoming greater than ever to address the basic health needs of the community and to achieve the sustainable development goals (SDG). A few studies in Ethiopia revealed that there was no waste segregation in most studied healthcare facilities. But, they were stored, transported, treated, and disposed wrongly $[26,27]$.

The proper healthcare waste management practice thought the best strategy to halt the spread of the infectious disease. However, there is a scanty study of the healthcare waste management practice in Amhara region. Hence, determining the healthcare waste management level and identifying its determinants is important to understand the gap and strengthen the existing strategies. Though the healthcare waste management practice was low in Ethiopia, there is also an unclear discrepancy between the public and private healthcare facilities. Therefore, the purpose of this study was to determine and compare the healthcare waste management practice level between private and public hospitals and to identify its associated factors in Bahir Dar city hospitals.

\section{Methods}

2.1. Study Design Area and Period. The institution-based cross-sectional study was conducted in Bahir Dar city hospitals from January 2016 to April 2017. Bahir Dar city is the capital city of Amhara National, Regional State [28], and is located about $570 \mathrm{~km}$ northwest of Addis Ababa. The city is bordered by Lake Tana in the north, South Gondar in the east and by West Gojjam in the south and west. The city has 9 subcities and 12 rural kebeles with a total population of 308,887 [28]. The city has one university, two referral hospitals, one district hospital, ten health centers, and two private hospitals. The number of healthcare workers in both public and private facilities is 823 . The study was conducted two public hospitals (one referral and one district) and two private hospitals.

2.2. Population. The source populations were all healthcare workers working in public and private hospitals in Bahir Dar city administration, while the study populations were selected healthcare workers in each healthcare facility working at least six months in the current hospital during the data collection period. Employed healthcare workers who are working less than six months in the current hospital were excluded.

2.3. Sample Size Determination. The sample size was calculated using the formula for two population proportions of the healthcare waste management practice level in public hospitals which is $p 1=40 \%$ [29] and in a private hospital is $p 2=55 \%$, with $1: 1$ ratio between private and public hospitals, power $=80 \%$, and confidence level $=95 \%$ (1.96). The formula can be seen as follows.

Accordingly, the required total sample size was 418. By considering a $10 \%$ nonresponse rate, the final sample size was 460 healthcare workers, 230 from private and 230 from public hospitals.

2.4. Sampling Procedures. For this study, all private and public hospitals in Bahir Dar city administration were included. Proportional to size allocation was used to select the sample from each private and public hospital and from each type of healthcare workers. The respondents were stratified by their department and profession, and then the study participants were selected from each group using systematic random sampling. The study included selected healthcare workers who were on duty at the time of the data collection period (Figure 1).

\subsection{Operational Definition}

2.5.1. Knowledge of Healthcare Waste Management. Healthcare workers who had a median score or above on the assessing questions regarding the knowledge of healthcare waste management were considered having a good knowledge of healthcare waste management. Otherwise, they were considered having poor knowledge of healthcare waste management.

2.5.2. Healthcare Waste Management Practice. Healthcare workers who had a mean score or above on the assessing questions regarding the healthcare waste management practice were considered having good healthcare waste management practice. Otherwise, they were considered as having poor healthcare waste management practice [30]. 

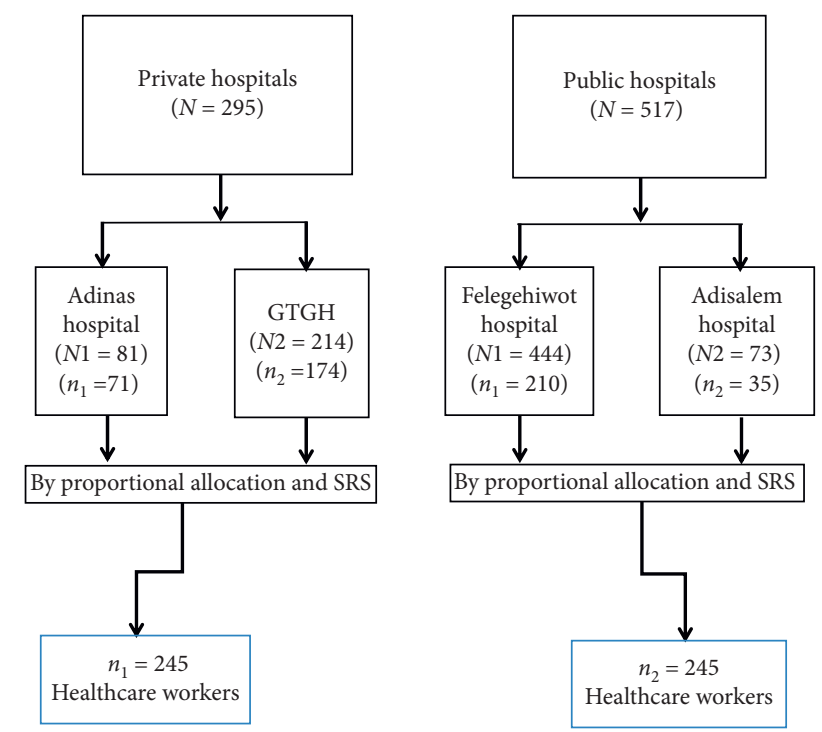

FIGURE 1: Schematic presentation of the sampling procedure on healthcare waste management, Bahir Dar city, 2017.

2.6. Data Collection Procedure. Data were collected by using a structured interviewer-administered Amharic (indigenous language) questionnaire, which was adapted from different literature [31-33]. The questionnaire is comprised of sociodemographic, knowledge, and healthcare institution related characteristics. The questionnaire was developed in English and translated to Amharic and then back to English to keep the consistency. Pretest was done by 40 healthcare workers (20 from private and 20 from the public) in Bure town. A one-day training was given for four data collectors (having a bachelor in nursing) and two supervisors (having a bachelor in public health). At the end of every data collection day, the supervisors examined each questionnaire and gave pertinent feedback to the data collectors.

2.7. Data Processing and Analysis. The collected data were checked for completeness and consistency. The data were cleaned, coded, and entered into EpiData software. Analysis was done using SPSS (version 20) software. Descriptive statistics were computed. Simple logistic regression model was used to identify the association between the explanatory variables and HCWMP. Adjusted odds ratio (AOR) with 95\% CI (confidence interval) was used to measure the strength of association between explanatory variables and the HCWMP. The model fitness was checked using Hosmer and Lemeshow goodness of fit $(p>0.05)$. A $p$ value $<0.2$ at bivariate analysis was considered for variables to be included in multivariable analysis. Backward logistic regression method was used, and variables with a $p$ value of $<0.05$ at multivariable analysis were considered as statistically significant predictors of HCWMP.

\section{Results}

3.1. Sociodemographic Information of Respondents. A total of 418 healthcare workers who are working in the private and public hospitals participated in the study and yielded a response of $90.9 \%$. The overall mean age of the respondents was $28.81( \pm 7.29)$ years. About $35.9 \%$ of the private hospital healthcare workers were diploma holders, while 151 (36.1\%) of the public hospital healthcare workers were degree graduate. More than one-third, 67 (34.9\%), of the private hospital healthcare workers and majority of the public hospital healthcare workers, $123(54.4 \%)$, were nurses. The majority of the private hospitals, 119 (62\%), and public hospitals, $136(60.2 \%)$, healthcare workers had a work experience of less than five years. More than two-thirds, 130 $(67.7 \%)$, of the respondents were from GAMBY hospital, and more than three-fourth $(85 \%)$ of the respondents were from Felegehiwot referral hospital (Table 1).

The study showed that $83(43.2 \%)$ of private hospital and $100(44.2 \%)$ of public hospital healthcare workers had training for the healthcare waste management, respectively. $161(83.9 \%)$ of private hospital and 179 (79.2\%) of public hospital healthcare workers had responded as there are the rules and regulations regarding healthcare waste management in their hospital. $133(69.3 \%)$ of private hospital and $140(61.9 \%)$ of public hospital healthcare workers had healthcare waste management team/committee (Table 2).

When assessing the knowledge level of healthcare workers regarding healthcare waste management practice using the composite index, 321 (72.2\%) of healthcare workers had good knowledge of healthcare waste management practice. 147 (76.6\%) of healthcare workers from private hospitals and 155 (68.6\%) from public hospitals had good knowledge of the healthcare waste management (Table 3 ).

The overall good healthcare waste management practice was $65.3 \%$ (95\% CI: 61, 70). More private healthcare workers, 79.2\% (95\% CI: 73, 85), had good healthcare waste management practice than public healthcare workers, $53.5 \%$ (95\% CI: 47, 60), respectively (Figure 2).

\subsection{Factors Associated with Healthcare Waste Management in} Bahir Dar City Hospitals. Multivariable logistic regression analysis was conducted to identify independent predictors of healthcare waste management practice of Bahir Dar city hospitals. On univariate logistic regression model ownership of the hospital, having legislation/regulation in the hospital, reporting system related to waste handling injury/accident, healthcare waste management committee, good knowledge of healthcare waste management, and guideline/ manual regarding the healthcare waste management was associated with healthcare waste management practice at $p$-value of less than 0.2 . However, in the multivariable logistic regression, healthcare facility ownership and having a healthcare waste management committee and a manual/guideline related to waste handling were significantly associated with healthcare waste management practice at $p$ value of less than 0.05 . 
TABLE 1: Sociodemographic characteristics of the respondents in Bahir Dar city hospitals, April 2017. Healthcare and institution related characteristics.

\begin{tabular}{|c|c|c|c|c|c|c|}
\hline \multirow{2}{*}{ Variable } & \multicolumn{2}{|c|}{ Private hospital } & \multicolumn{2}{|c|}{ Public hospital } & \multicolumn{2}{|c|}{ Total } \\
\hline & Number & Percent & Number & Percent & Number & Percent \\
\hline \multicolumn{7}{|l|}{ Age (in years) } \\
\hline $18-25$ & 92 & 47.9 & 120 & 53.1 & 212 & 50.7 \\
\hline $26-35$ & 75 & 39.1 & 74 & 32.7 & 149 & 35.6 \\
\hline $36-45$ & 16 & 8.3 & 20 & 8.8 & 36 & 8.6 \\
\hline 46 and above & 9 & 4.7 & 12 & 5.3 & 21 & 5.0 \\
\hline \multicolumn{7}{|l|}{ Sex } \\
\hline Male & 90 & 46.9 & 94 & 41.6 & 184 & 44.0 \\
\hline Female & 102 & 53.1 & 132 & 58.4 & 234 & 56.0 \\
\hline \multicolumn{7}{|l|}{ Educational status } \\
\hline Secondary school and below & 38 & 19.8 & 23 & 10.2 & 61 & 14.6 \\
\hline Certificate & 6 & 3.1 & 1 & .4 & 7 & 1.7 \\
\hline Diploma & 69 & 35.9 & 74 & 32.7 & 143 & 34.2 \\
\hline Degree & 48 & 25.0 & 103 & 45.6 & 151 & 36.1 \\
\hline Master & 31 & 16.1 & 25 & 11.1 & 56 & 13.4 \\
\hline \multicolumn{7}{|l|}{ Profession } \\
\hline Health officer & 4 & 2.1 & - & - & 4 & 1.0 \\
\hline Physician & 23 & 12.0 & 19 & 8.4 & 42 & 10.0 \\
\hline Laboratory & 21 & 10.9 & 16 & 7.1 & 37 & 8.9 \\
\hline Pharmacist & 18 & 9.4 & 17 & 7.5 & 35 & 8.4 \\
\hline Radiographer & 7 & 3.6 & 7 & 3.1 & 14 & 3.3 \\
\hline Midwifery & 8 & 4.2 & 16 & 7.1 & 24 & 5.7 \\
\hline Cleaner & 33 & 17.2 & 23 & 10.2 & 56 & 13.4 \\
\hline Environmental health officer & 2 & 1.0 & 2 & .9 & 4 & 1.0 \\
\hline Nurse & 67 & 34.9 & 123 & 54.4 & 190 & 45.5 \\
\hline Laundry workers & 9 & 4.7 & 3 & 1.3 & 12 & 2.9 \\
\hline \multicolumn{7}{|l|}{ Work experience (in years) } \\
\hline $0-5$ & 119 & 62.0 & 136 & 60.2 & 255 & 61.0 \\
\hline $6-10$ & 41 & 21.4 & 60 & 26.5 & 101 & 24.2 \\
\hline$\geq 11$ & 32 & 16.7 & 30 & 13.3 & 62 & 14.8 \\
\hline \multicolumn{7}{|l|}{ Respondents affiliation (hospital) } \\
\hline Adinas & 62 & 32.3 & - & - & 62 & 14.8 \\
\hline GAMBY & 130 & 67.7 & - & - & 130 & 31.1 \\
\hline Felegehiwot & - & - & 193 & 85.4 & 193 & 46.2 \\
\hline Addisalem & - & - & 33 & 14.6 & 33 & 7.9 \\
\hline
\end{tabular}

TABLE 2: Healthcare and institution related characteristics in public and private hospitals in Bahir Dar city, April 2017. Knowledge-related characteristics.

\begin{tabular}{|c|c|c|c|c|c|c|}
\hline \multirow{2}{*}{ Variables } & \multicolumn{2}{|c|}{ Private hospitals } & \multicolumn{2}{|c|}{ Public hospitals } & \multicolumn{2}{|c|}{ Total } \\
\hline & Number & Percent & Number & Percent & Number & Percent \\
\hline \multicolumn{7}{|c|}{ Have you taken any training on healthcare waste management? } \\
\hline Yes & 83 & 43.2 & 100 & 44.2 & 183 & 43.8 \\
\hline No & 109 & 56.8 & 126 & 55.8 & 235 & 56.2 \\
\hline \multicolumn{7}{|c|}{ Are there any healthcare waste management rules and regulations in your facility? } \\
\hline Yes & 161 & 83.9 & 179 & 79.2 & 340 & 81.3 \\
\hline No & 12 & 6.3 & 16 & 7.1 & 28 & 6.7 \\
\hline Do not know & 19 & 9.9 & 31 & 13.7 & 50 & 12.0 \\
\hline \multicolumn{7}{|c|}{ Does your healthcare facility have healthcare waste management system? } \\
\hline Yes & 103 & 53.6 & 113 & 50.0 & 216 & 51.7 \\
\hline No & 89 & 46.4 & 113 & 50.0 & 202 & 48.3 \\
\hline \multicolumn{7}{|c|}{ Does your healthcare facility have a waste management team/committee? } \\
\hline Yes & 133 & 69.3 & 140 & 61.9 & 273 & 65.3 \\
\hline No & 59 & 30.7 & 86 & 38.1 & 145 & 34.7 \\
\hline \multicolumn{7}{|c|}{ Is your healthcare facility waste management team functional? } \\
\hline Yes & 91 & 68.4 & 84 & 60.0 & 175 & 64.1 \\
\hline No & 42 & 31.6 & 56 & 40.0 & 98 & 35.9 \\
\hline
\end{tabular}


TABLE 2: Continued.

\begin{tabular}{|c|c|c|c|c|c|c|}
\hline \multirow{2}{*}{ Variables } & \multicolumn{2}{|c|}{ Private hospitals } & \multicolumn{2}{|c|}{ Public hospitals } & \multicolumn{2}{|c|}{ Total } \\
\hline & Number & Percent & Number & Percent & Number & Percent \\
\hline \multicolumn{7}{|c|}{ Is there any guideline document on healthcare waste management you are aware of? } \\
\hline Yes & 172 & 89.6 & 194 & 85.8 & 366 & 87.6 \\
\hline No & 20 & 10.4 & 32 & 14.2 & 52 & 12.4 \\
\hline \multicolumn{7}{|c|}{ Is there any regular healthcare waste management training for all staff? } \\
\hline Yes & 23 & 12.0 & 31 & 13.7 & 54 & 12.9 \\
\hline No & 169 & 88.0 & 195 & 86.3 & 364 & 87.1 \\
\hline \multicolumn{7}{|c|}{ Is there enough personal protective equipment and other supplies for HCWM? } \\
\hline Yes & 134 & 69.8 & 151 & 66.8 & 285 & 68.2 \\
\hline No & 58 & 30.2 & 75 & 33.2 & 133 & 31.8 \\
\hline \multicolumn{7}{|c|}{ Have you ever experienced waste handling related injury in the last 12 months? } \\
\hline Yes & 23 & 12.0 & 31 & 13.7 & 54 & 12.9 \\
\hline No & 169 & 88.0 & 195 & 86.3 & 364 & 87.1 \\
\hline
\end{tabular}

TABle 3: Knowledge-related characteristics in public and private hospitals in Bahir Dar city, April 2017. The prevalence of healthcare waste management practice between private and public hospitals.

\begin{tabular}{|c|c|c|c|c|c|c|}
\hline \multirow{2}{*}{ Variables } & \multicolumn{2}{|c|}{ Private } & \multicolumn{2}{|c|}{ Government } & \multicolumn{2}{|c|}{ Total } \\
\hline & Number & Percent & Number & Percent & Number & Percent \\
\hline \multicolumn{7}{|c|}{ Improper color-coding segregation of healthcare waste increases the risk of injury } \\
\hline Yes & 188 & 97.9 & 220 & 97.3 & 188 & 97.9 \\
\hline No & 4 & 2.1 & 6 & 2.7 & 4 & 2.1 \\
\hline \multicolumn{7}{|c|}{ Improper color-coding segregation of healthcare waste increases the amount of infectious healthcare waste } \\
\hline Yes & 184 & 95.8 & 217 & 96.0 & 401 & 95.9 \\
\hline No & 8 & 4.2 & 9 & 4.0 & 17 & 4.1 \\
\hline \multicolumn{7}{|c|}{ Improper color-coding segregation of healthcare waste contributes to improper healthcare waste disposal } \\
\hline Yes & 155 & 80.7 & 169 & 74.8 & 324 & 77.5 \\
\hline No & 37 & 19.3 & 57 & 25.2 & 94 & 22.5 \\
\hline \multicolumn{7}{|c|}{ Mixing of infectious waste with noninfectious waste contributes to disease transmission } \\
\hline Yes & 147 & 76.6 & 171 & 75.7 & 318 & 76.1 \\
\hline No & 45 & 23.4 & 55 & 24.3 & 100 & 23.9 \\
\hline \multicolumn{7}{|c|}{ Improper healthcare waste disposal contributes to disease transmission } \\
\hline Yes & $177^{1}$ & 92.2 & 220 & 97.3 & 397 & 95.0 \\
\hline No & 15 & 7.8 & 6 & 2.7 & 21 & 5.0 \\
\hline \multicolumn{7}{|c|}{ Disposing of HCW without treatment contributes to disease transmission } \\
\hline Yes & 28 & 14.6 & 42 & 18.6 & 70 & 16.7 \\
\hline No & 164 & 85.4 & 184 & 81.4 & 348 & 83.3 \\
\hline \multicolumn{7}{|c|}{ HIV can be transmitted from unsafe healthcare waste management practice } \\
\hline Yes & 179 & 93.2 & 211 & 93.4 & 390 & 93.3 \\
\hline No & 13 & 6.8 & 15 & 6.6 & 28 & 6.7 \\
\hline \multicolumn{7}{|c|}{ Hepatitis $B$ and $C$ can be transmitted from unsafe healthcare waste management practice } \\
\hline Yes & 171 & 89.1 & 193 & 85.4 & 364 & 87.1 \\
\hline No & 21 & 10.9 & 33 & 14.6 & 54 & 12.9 \\
\hline \multicolumn{7}{|c|}{ Hepatitis A can be transmitted from unsafe healthcare waste management practice } \\
\hline Yes & 140 & 72.9 & 128 & 56.6 & 268 & 64.1 \\
\hline No & 52 & 27.1 & 98 & 43.4 & 150 & 35.9 \\
\hline \multicolumn{7}{|c|}{ Ebola can be transmitted from unsafe healthcare waste management practice } \\
\hline Yes & 159 & 82.8 & 162 & 71.7 & 321 & 76.8 \\
\hline No & 33 & 17.2 & 64 & 28.3 & 97 & 23.2 \\
\hline \multicolumn{7}{|c|}{ Knowledge score } \\
\hline Good & 147 & 76.6 & 155 & 68.6 & 302 & 72.2 \\
\hline Poor & 45 & 23.4 & 71 & 31.4 & 116 & 27.8 \\
\hline
\end{tabular}

Accordingly, for healthcare workers working in a private hospital, the odds ratio of healthcare waste management practice was about two $(\mathrm{AOR}=2.89,95 \% \mathrm{CI}: 1.85,4.51)$ times higher compared to those working in public hospitals. Similarly, for healthcare workers who had a committee of healthcare waste management, the odds ratio of healthcare 


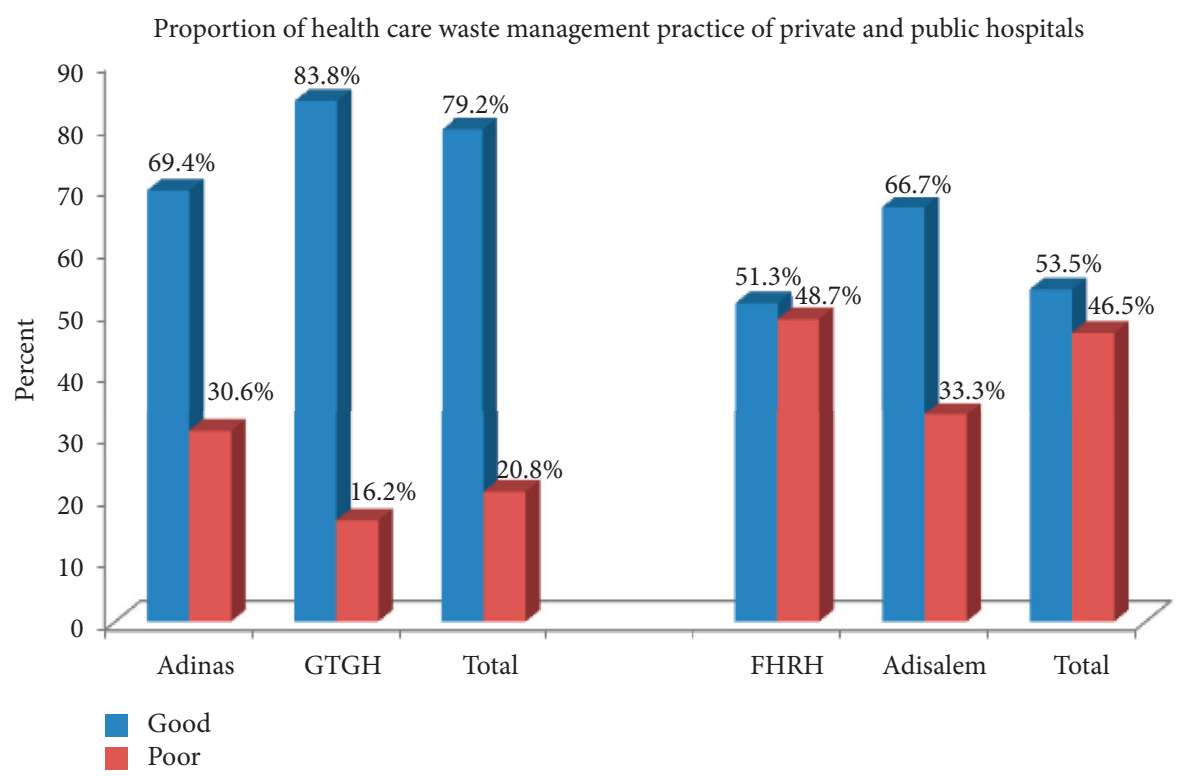

FIGURE 2: The proportion of healthcare waste management practice between private and public hospitals.

waste management practice was about $1.75(\mathrm{AOR}=112$, 95\% CI: 1.12, 2.72) times higher compared to those who did not have one. It is also revealed that, in the hospitals which had a manual/guideline related to waste handling, the odds ratio of healthcare waste management practice was about two times $(\mathrm{AOR}=2.06,95 \% \mathrm{CI}: 1.152,3.684)$ higher compared to those who did not have one (Table 4).

Multivariable logistic regression analysis was conducted to identify independent predictors of healthcare waste management practice of Bahir Dar city private hospitals. In the univariate logistic regression, sex, having a healthcare waste management committee, functional healthcare waste management committee, reporting system related to waste handling injury/accident, and HCWMP included in the job description were associated with healthcare waste management practice at $p$-value of less than 0.2 . However, in the multivariable logistic regression, only sex and having a functional healthcare waste management committee were significantly associated with healthcare waste management practice at $p$-value of less than 0.05 .

For male healthcare workers working in a private hospital, the odds ratio of healthcare waste management practice was about $\operatorname{six}(\mathrm{AOR}=6.43,95 \% \mathrm{CI}: 1.82,22.77)$ times higher compared to female healthcare workers. Similarly, for healthcare workers who have functional healthcare waste management committee, the odds ratio of healthcare waste management practice was about six $(\mathrm{AOR}=6.47,95 \% \mathrm{CI}: 1.93,21.76)$ times higher compared to those who did not have one (Table 5).

Multivariable logistic regression analysis was conducted to identify independent predictors of healthcare waste management practice of Bahir Dar city private hospitals. In the univariate logistic regression, sex, having rules and regulation/legislation on HCWM, a manual/guideline related to waste handling, and a healthcare waste management committee, and HCWMP included in the job description
TABLE 4: Factors associated with healthcare waste management (HCWM) practice in Bahir Dar city hospitals, $2017 \quad(n=418)$. Factors associated with healthcare waste management in private hospitals.

\begin{tabular}{|c|c|c|c|c|c|}
\hline \multirow[t]{2}{*}{ Variables } & \multicolumn{2}{|c|}{$\begin{array}{l}\text { HCWM } \\
\text { practice }\end{array}$} & \multirow[t]{2}{*}{$\mathrm{COR}$ at $95 \% \mathrm{CI}$} & \multirow{2}{*}{$\begin{array}{c}\mathrm{AOR} \text { at } 95 \% \\
\mathrm{CI}\end{array}$} & \multirow[t]{2}{*}{$p$ value } \\
\hline & Good & Poor & & & \\
\hline \multicolumn{6}{|c|}{ Ownership of the hospital } \\
\hline Private & 152 & 40 & $\begin{array}{c}3.298(2.133 \\
5.097)\end{array}$ & $\begin{array}{c}2.89(1.85, \\
4.51)\end{array}$ & 0.0001 \\
\hline Public & 121 & 105 & 1.00 & 1.00 & \\
\hline \multicolumn{6}{|c|}{ Rules and regulations/legislations on HCWM in your facility } \\
\hline Yes & 231 & 109 & $\begin{array}{c}1.817(1.102 \\
2.995)\end{array}$ & $\begin{array}{c}1.28(0.702 \\
2.326)\end{array}$ & 0.423 \\
\hline No & 42 & 36 & 1.00 & 1.00 & \\
\hline \multicolumn{6}{|c|}{ Healthcare waste management committee } \\
\hline Yes & 193 & 80 & $\begin{array}{c}1.96(1.29 \\
2.978)\end{array}$ & $\begin{array}{c}1.75(1.123 \\
2.72)\end{array}$ & 0.013 \\
\hline No & 80 & 65 & 1.00 & 1.00 & \\
\hline \multicolumn{6}{|c|}{ Manual/guideline related to waste handling } \\
\hline Yes & 242 & 111 & $\begin{array}{c}2.39(1.39 \\
4.087)\end{array}$ & $\begin{array}{c}2.06(1.152, \\
3.684)\end{array}$ & 0.015 \\
\hline No & 31 & 34 & 1.00 & 1.00 & \\
\hline \multicolumn{6}{|c|}{ Having a reporting system related to waste handling injury/accident } \\
\hline Yes & 180 & 82 & $1.59(1.05,2.41)$ & $\begin{array}{c}0.89(0.532 \\
1.508)\end{array}$ & 0.678 \\
\hline No & 87 & 63 & 1.00 & 1.00 & \\
\hline \multicolumn{6}{|c|}{ Knowledge of healthcare waste management } \\
\hline Good & 207 & 95 & $\begin{array}{c}\text { 1.651(1.06, } \\
2.56)\end{array}$ & $\begin{array}{c}1.315(0.814 \\
2.123)\end{array}$ & 0.263 \\
\hline Poor & 66 & 50 & 1.00 & 1.00 & \\
\hline
\end{tabular}

were associated with healthcare waste management practice at $p$-value of less than 0.2 . However, in the multivariable logistic regression, only having a manual/guideline related to waste handling and healthcare waste management 
TABLE 5: Factors associated with healthcare waste management (HCWM) practice in Bahir Dar city private hospitals, 2017 $(n=192)$. Factors associated with healthcare waste management in public hospitals.

\begin{tabular}{|c|c|c|c|c|c|}
\hline \multirow[t]{2}{*}{ Variables } & \multicolumn{2}{|c|}{$\begin{array}{l}\text { HCWM } \\
\text { practice }\end{array}$} & \multirow{2}{*}{$\begin{array}{c}\text { COR at } 95 \% \\
\text { CI }\end{array}$} & \multirow[t]{2}{*}{$\mathrm{AOR}$ at $95 \% \mathrm{CI}$} & \multirow[t]{2}{*}{$p$ value } \\
\hline & Good & Poor & & & \\
\hline \multicolumn{6}{|l|}{ Sex } \\
\hline Male & 74 & 15 & $\begin{array}{c}1.623(1.794 \\
3.318)\end{array}$ & $\begin{array}{c}6.43(1.817 \\
22.769)\end{array}$ & 0.004 \\
\hline Female & 77 & 25 & 1.00 & 1.00 & \\
\hline
\end{tabular}

Having a healthcare waste management committee

\begin{tabular}{lccccc} 
Yes & 109 & 24 & $1.690(1.819$, & $1.32(0.23$, & 0.58 \\
& & & $3.487)$ & $2.72)$ & \\
No & 43 & 16 & 1.00 & 1.00 & \\
\hline
\end{tabular}

Having a functional healthcare waste management committee

\begin{tabular}{llcccc} 
Yes & 69 & 11 & $2.039(1.235$, & $\mathbf{6 . 4 7 3}(\mathbf{1 . 9 3}$, & $\mathbf{0 . 0 0 3}$ \\
No & 40 & 13 & $4.976)$ & $\mathbf{2 1 . 7 6 )}$ & \\
\hline
\end{tabular}

Having a reporting system related to waste handling injury/accident $\begin{array}{lllccc}\text { Yes } & 105 & 26 & \begin{array}{c}1.379(1.656, \\ 2.900)\end{array} & \begin{array}{c}0.524(0.124, \\ 2.217)\end{array} & 0.380\end{array}$

$\begin{array}{lllll}\text { No } & 41 & 14 & 1.00 & 1.00\end{array}$

Healthcare waste management included in the healthcare worker's job description

\begin{tabular}{llcccc} 
Yes & 89 & 25 & $1.903(1.37$, & $1.018(0.306$, & 0.977 \\
& & & $3.867)$ & $3.380)$ & \\
No & 57 & 15 & 1.00 & 1.00 & \\
\hline
\end{tabular}

committee were significantly associated with healthcare waste management practice at $p$-value of less than 0.05 .

Accordingly, for healthcare workers who had healthcare waste management committee, the odds ratio of healthcare waste management practice was about two $(\mathrm{AOR}=1.80$, $95 \%$ CI: $1.03,3.15)$ times higher compared to those who did not have one. Similarly, for healthcare workers who have a manual/guideline related to waste handling, the odds ratio of healthcare waste management practice was about two $(\mathrm{AOR}=2.43,95 \% \mathrm{CI}: 1.20,4.91)$ times higher compared to those who did not have one (Table 6).

\section{Discussion}

The World Health Organization has organized a healthcare waste management guideline to warrant safe healthcare waste management. Furthermore, a Quality and Standard Authority of Ethiopia (in 2004) and the Federal Ministry of Health of Ethiopia (in 1997) had also prepared a guideline on healthcare waste handling and disposal within healthcare facilities [34].

This study determined the level of healthcare waste management practices among private and public hospitals and identified the associated factors in the Bahir Dar city. More private hospitals (79.2\%) had a good healthcare waste management practice than public hospitals (53.5\%). Male healthcare workers, having a functional healthcare waste management committee in private hospitals, and availability of healthcare waste management manual/guideline and
TABLE 6: Factors associated with healthcare waste management (HCWM) practice in Bahir Dar city public hospitals, 2017 $(n=226)$.

\begin{tabular}{lccccc}
\hline Variables & $\begin{array}{c}\text { HCWM } \\
\text { practice } \\
\text { Good Poor }\end{array}$ & $\begin{array}{c}\text { COR at 95\% } \\
\text { CI }\end{array}$ & AOR at 95\% CI & $p$ value \\
\hline Sex & & & $0.584(0.342$, & $0.668(0.384$, & 0.152 \\
Male & 43 & 51 & $0.996)$ & $1.160)$ & \\
Female & 78 & 54 & 1.00 & 1.00 & \\
\hline
\end{tabular}

Availability of rules and regulations/legislations on HCWM in your facility

\begin{tabular}{lccccc} 
Yes & 104 & 75 & $2.447(1.259$, & $1.276(0.562$, & 0.561 \\
No & 17 & 30 & $1.758)$ & $2.897)$ & \\
\hline
\end{tabular}

Availability of a manual/guideline related to waste handling

$\begin{array}{lccccc}\text { Yes } & 106 & 75 & 2.827(1.422, & \mathbf{2 . 4 3 0 ( 1 . 2 0 3 ,} & \mathbf{0 . 0 1 3} \\ & & & 5.617) & \mathbf{4 . 9 0 8 )} & \\ \text { No } & 15 & 30 & 1.00 & 1.00 & \end{array}$

\begin{tabular}{lccccc}
\hline \multicolumn{5}{c}{ Availability of a healthcare waste management committee } \\
Yes & 84 & 56 & $1.986(1.152$, & $\mathbf{1 . 8 0 1}(\mathbf{1 . 0 2 9}$, & $\mathbf{0 . 0 3 9}$ \\
No & 37 & 49 & $3.424)$ & $\mathbf{3 . 1 5 2})$ & \\
\hline
\end{tabular}

Healthcare waste management included in the job description

\begin{tabular}{lllccc} 
Yes & 86 & 56 & $2.106(1.215$, & $1.363(0.723$, & 0.338 \\
& & & $3.652)$ & $2.569)$ & \\
No & 35 & 48 & 1.00 & 1.00 & \\
\hline
\end{tabular}

committee in public hospitals were significantly associated with healthcare waste management practice.

The current study revealed that 79.2\% (95\% CI: 73,85$)$ of private hospitals had good healthcare waste management practice. The result of this study was higher than other studies on private hospitals in Addis Ababa, Ethiopia (40.48\%) [35], Bahawalpur City, Pakistan (41.6\%) [36], and Nigeria (62\%) [37]. The possible justification for this discrepancy could be the variation of the study time. The former study was carried out recently, where the healthcare management practice is improving due to the increased awareness and attitude of the healthcare workers via IEC (behavioral change communication). The other possible justification for this variation could be using different healthcare waste management practice assessing tools across different studies.

The study also determined the healthcare waste management practice of public hospitals, which was 53.5\% (95\% CI: 47, 60). This finding was consistent with the findings of other studies on public hospitals in Addis Ababa 57.37\% [38] (59.22\%) [35] and Bangladesh (54\%) [39]. However, the result of this study was lower than other studies done in Bahawalpur City, Pakistan (66.6\%) [36] and Nigeria (98.4\%) [40]. This might be due to the difference in healthcare system/policy and the healthcare planners towards the healthcare waste management practice. This finding might also be justified as a result of the ignorance of the healthcare workers regarding healthcare waste management practice.

In contrast, the result of the current study was higher than other studies on Adama Hospital, Ethiopia, 34.9\% [41], 
Gondar Town, Ethiopia, 31.5\% and 46.30\% [42, 43], Nigeria, 42.1\% [44], Burundi, 29.5\% [45], and Bangladesh, 36.03\% [46]. The possible reason for this variation could be the difference in the study period, study area, and sample size used.

The current study also identified various factors associated with healthcare waste management practices. Ownership of the hospital was also correlated with healthcare management practices. Healthcare workers working at private hospitals were more likely to be having good healthcare waste management practice than public hospital healthcare workers. This could be explained by the tendency that the private hospitals are more business-oriented and they need to attract the attention of the clients or patients with their clear and neat compound. They are also supposed to engage in the free market and should have the current standard equipment and this might allow good practice of healthcare waste management. Also, in the private hospitals, the community might expect safer and infection-free services as a result, and this might help them to best practice healthcare waste management.

This study has revealed that hospitals which had a healthcare waste management committee and manual/ guideline related to healthcare waste handling had about 2fold good healthcare waste management practice compared to those which did not have any. This finding was also observed in this study of public hospitals. This could be due to the fact that hospitals staff was advised on the importance of healthcare waste management practice by the committee members or by reading the manuals/guidelines of the healthcare waste management protocol.

Male sex was one of the explanatory variables statistically associated with good healthcare waste management practice at the private health facility, but not in the public health facilities.

The other significant predictor of healthcare waste management practice in private hospitals was having a functional healthcare waste management committee. Privately owned hospitals, which had functional healthcare waste management committee, were correlated with good healthcare waste management practices. However, no correlation was found at public hospitals. This might be due to the fact that healthcare workers in private hospitals have strict follow-up and supervision and evaluation programs, which in fact allow the formed committee to be well functional in practicing healthcare waste management, and as a result, private hospitals could have a better experience of healthcare waste management practice.

4.1. Strength and Limitation of the Study. Even though this study has provided valuable evidence regarding the level of healthcare waste management practice and the possible associated factors, it could not avoid the chicken-egg dilemma. There was also a challenge of having standard measuring cut-points for healthcare waste management practice. It was also difficult to collect the previously published articles related to healthcare waste management to make comparisons, particularly, in the currently identified predictors.

\section{Conclusion}

The study revealed that there was a great discrepancy between private and public hospitals in healthcare waste management practice. More private hospitals, 79.2\% (95\% CI: 73,85$)$, had a good healthcare waste management practice compared to public hospitals, 53.5\% (95\%CI: 47, 60). Male healthcare workers and having functional healthcare waste management committee were significantly associated with healthcare waste management practice in private hospitals, whereas having a healthcare waste management committee and a manual/guideline on healthcare waste management was significantly associated with healthcare waste management practice in public hospitals.

5.1. Recommendations. The Amhara Regional Health $\mathrm{Bu}-$ reau should do the following:

(1) Focus on strategies regarding the healthcare waste management committee establishment in each hospital and the provision of enough healthcare waste management related manuals/guidelines to the hospitals

(2) Organize short-term training and an orientation program on healthcare waste management for their staff

The private hospitals should do the following:

(1) Share their best experiences on how they are practicing the healthcare waste management with the public hospitals

(2) Train the public hospital healthcare workers on how the healthcare waste management committee is functioning

(3) Train their female healthcare workers to have a better healthcare waste management practice
Abbreviations
AOR: Adjusted odds ratio
BSc: $\quad$ Bachelor of science
COR: Crude odds ratio
EpiData: Epidemiological data
IEC: Information Education and Communication
MOH: Ministry of Health
MPH: Master of public health
SPSS: $\quad$ Statistical Package for Social Sciences.

\section{Data Availability}

The data are available from the corresponding author upon justified request.

\section{Ethical Approval}

Ethical approval was obtained from institutional review board of Bahir Dar university. The approval letter was 
obtained from the Amhara Public Health Institute. The institutional review board approved the procedure for verbal consent as the study is not sensitive and has no privacy issues; rather it assessed the practice of healthcare waste management at the hospital level. The objective of the study was clarified, and informed verbal consent was received from the study participants to confirm their willingness. The respondents were notified that they have the right to refuse or terminate the study at any point of the interview. Because the authors obtained verbal consent, documentation of consent was not required. However, the information provided by each respondent was kept confidential in a secure place.

\section{Conflicts of Interest}

The authors declare that they have no conflicts of interest.

\section{Authors' Contributions}

DMA, TET, YMG, and GMB conceived the study, approved the proposal with extensive revisions, participated in the data analysis, and had written the manuscript. The authors read and approved the final manuscript.

\section{Acknowledgments}

The authors would like to thank the data collectors, supervisors, study participants, and Bahir Dar private and public hospital medical directors for their cooperation and help during the data collection.

\section{References}

[1] M. E. Birpınar, M. S. Bilgili, and T. Erdoğan, "Medical waste management in Turkey: a case study of Istanbul," Waste Management, vol. 29, no. 1, pp. 445-448, 2009.

[2] M. Chaerul, M. Tanaka, and A. V. Shekdar, "A system dynamics approach for hospital waste management," Waste Management, vol. 28, no. 2, pp. 442-449, 2008.

[3] WHO, Preparation of National Health-Care Waste Management Plans in Sub-Saharan Countries, WHO, Geneva, Switzerland, 2013.

[4] WHO, AIDE-MEMOIRE for a National Strategy for HealthCare Waste Management, WHO, Geneva, Switzerland, 2015.

[5] N. Marinković, K. Vitale, N. Janev Holcer, A. Džakula, and T. Pavić, "Management of hazardous medical waste in Croatia," Waste Management (New York, N.Y.), vol. 28, no. 6, pp. 1049-1056, 2008.

[6] A. Prüss-Üstün, E. Giroult, P. Rushbrook, and W. H. Organization, Safe Management of Wastes from HealthCare Activities, World Health Organization, Geneva, Switzerland, 1999.

[7] M. H. Dehghani, H. D. Ahrami, R. Nabizadeh, Z. Heidarinejad, and A. Zarei, "Medical waste generation and management in medical clinics in South of Iran," MethodsX, vol. 6, pp. 727-733, 2019.

[8] M. Tsakona, E. Anagnostopoulou, and E. Gidarakos, "Hospital waste management and toxicity evaluation: a case study," Waste Management, vol. 27, no. 7, pp. 912-920, 2007.

[9] WHO, Safe Healthcare Waste Management: Policy Paper, World Health Organization, Geneva, Switzerland, 2004.
[10] M. A. Patwary, W. T. O’Hare, G. Street, K. Maudood Elahi, S. S. Hossain, and M. H. Sarker, "Quantitative assessment of medical waste generation in the capital city of Bangladesh," Waste Management, vol. 29, no. 8, pp. 2392-2397, 2009.

[11] S. A. Tamplin, D. Davidson, B. Powis, and Z. O'Leary, "Issues and options for the safe destruction and disposal of used injection materials," Waste Management, vol. 25, no. 6, pp. 655-665, 2005.

[12] M. Sawalem, "Hospital waste management in Libya: a case study," Waste Management, vol. 29, no. 13, pp. 70-75, 2009.

[13] Y.-C. Jang, C. Lee, O.-S. Yoon, and H. Kim, "Medical waste management in Korea," Journal of Environmental Management, vol. 80, no. 2, pp. 7-15, 2006.

[14] WHO, Healthcare Waste Management, World Health Organization, Geneva, Switzerland, 2005.

[15] L. F. Diaz, G. M. Savage, and L. L. Eggerth, "Alternatives for the treatment and disposal of healthcare wastes in developing countries," Waste Management, vol. 25, no. 6, pp. 626-637, 2005.

[16] B.-K. Lee, M. J. Ellenbecker, and R. Moure-Ersaso, "Alternatives for treatment and disposal cost reduction of regulated medical wastes," Waste Management, vol. 24, no. 2, pp. 143-151, 2004.

[17] F. Abdulla, H. Abu Qdais, and A. Rabi, "Site investigation on medical waste management practices in northern Jordan," Waste Management, vol. 28, no. 2, pp. 450-458, 2008.

[18] C. E. Da Silva, A. E. Hoppe, M. M. Ravanello, and N. Mello, "Medical wastes management in the south of Brazil," Waste Management, vol. 25, no. 6, pp. 600-605, 2005.

[19] M. Askarian, M. Vakili, and G. Kabir, "Results of a hospital waste survey in private hospitals in Fars Province, Iran," Waste Management, vol. 24, no. 4, pp. 347-352, 2004.

[20] E. Shinee, E. Gombojav, A. Nishimura, N. Hamajima, and K. Ito, "Healthcare waste management in the capital city of Mongolia," Waste Management, vol. 28, no. 2, pp. 435-441, 2008.

[21] E. Insa, M. Zamorano, and R. López, "Critical review of medical waste legislation in Spain," Resources, Conservation and Recycling, vol. 54, no. 12, pp. 1048-1059, 2010.

[22] E. Bazrafshan and F. Kord Mostafapoor, "Survey of medical waste characterization and management in Iran: a case study of Sistan and Baluchestan Province," Waste Management \& Research, vol. 29, no. 4, pp. 442-450, 2011.

[23] A. Coker, A. Sangodoyin, M. Sridhar, C. Booth, P. Olomolaiye, and F. Hammond, "Medical waste management in Ibadan, Nigeria: obstacles and prospects," Waste Management, vol. 29, no. 2, pp. 804-811, 2009.

[24] G. M. A. Mostafa, M. M. Shazly, and W. I. Sherief, "Development of a waste management protocol based on assessment of knowledge and practice of healthcare personnel in surgical departments," Waste Management, vol. 29, no. 1, pp. 430-439, 2009.

[25] M. A. Patwary, W. T. O'Hare, and M. H. Sarker, “Assessment of occupational and environmental safety associated with medical waste disposal in developing countries: a qualitative approach," Safety Science, vol. 49, no. 8-9, pp. 1200-1207, 2011.

[26] I. D. Haylamicheal, M. A. Dalvie, B. D. Yirsaw, and H. A. Zegeye, "Assessing the management of healthcare waste in Hawassa city, Ethiopia," Waste Management \& Research, vol. 29, no. 8, pp. 854-862, 2011.

[27] S. D. Yimer, "Healthcare waste management in Ethiopia," IOHA Pilansberg, vol. 29, 2005. 
[28] Amhara National, Regional State Bureau of Finance and Economic Development, 2016/2017.

[29] MaHAaCA, "Health care waste management directive," 2005.

[30] S. T. Wafula, J. Musiime, and F. Oporia, "Health care waste management among health workers and associated factors in primary health care facilities in Kampala City, Uganda: a crosssectional study," BMC Public Health, vol. 19, no. 1, p. 203, 2019.

[31] Framework for Conducting a Self-Assessment of National Progress in Injection Waste Management, Version -1, 2008.

[32] WHO Secretariat of the Basel Convention, Preparation of National Health-Care Waste Management Plans in SubSaharan Countries Guideline, WHO, Geneva, Switzerland.

[33] WHO, Health Care Waste Management Rapid Assessment Tool for Country Level, WHO, Geneva, Switzerland.

[34] EFMoH, Infection Prevention Guidelines for Healthcare Facilities in Ethiopia, Disease Prevention and Control Department, Addis Ababa, Ethiopia, 2004.

[35] M. K. Debere, K. A. Gelaye, A. G. Alamdo, and Z. M. Trifa, "Assessment of the health care waste generation rates and its management system in hospitals of Addis Ababa, Ethiopia, 2011," BMC Public Health, vol. 13, no. 28, 2013.

[36] S. Badar, "Health care waste management practices in public and private sector hospitals," Journal of Rawalpindi Medical College, vol. 18, no. 1, pp. 145-147, 2014.

[37] A. O. Tope, A. O. Olufemi, A. B. Kehinde, and F. F. Omolola, "Healthcare waste management practices and risk perception of healthcare workers in private healthcare facilities in an urban community in Nigeria," African Journal of Environmental Science and Technology, vol. 12, no. 9, pp. 305-311, 2018.

[38] M. L. Tadesse and A. Kumie, "Healthcare waste generation and management practice in government health centers of Addis Ababa, Ethiopia," BMC Public Health, vol. 14, no. 1, p. 1221, 2014.

[39] M. M. Hasan and M. H. Rahman, "Assessment of healthcare waste management paradigms and its suitable treatment alternative: a case study," Journal of Environmental and Public Health, vol. 2018, 2018.

[40] A.B. Umar and M. N Yahaya, "Hospital waste management practices: a case study of primary health care centers, in Fagge Local Government Area, Kano State, Nigeria," IOSR Journal of Nursing and Health Science, vol. 3, no. 6, pp. 26-33, 2014.

[41] S. F. Hayleeyesus and W. Cherinete, "Healthcare waste generation and management in public healthcare facilities in Adama, Ethiopia," Journal of Health and Pollution, vol. 6, no. 10, pp. 64-73, 2016.

[42] A. Muluken, G. Haimanot, and M. Mesafint, "Healthcare waste management practices among healthcare workers in healthcare facilities of Gondar Town, Northwest Ethiopia," Health Science Journal, vol. 7, no. 3, 2013.

[43] A. Mesfin, W. Worku, and Z. Gizaw, "Assessment of health care waste segregation practice and associated factors of health care workers in Gondar University Hospital, North West Ethiopia," Universal Journal of Public Health, vol. 2, no. 7, pp. 201-207, 2013.

[44] N. Ngwuluka, N. Ochekpe, P. Odumosu, and S. A. John, "Waste management in healthcare establishments within Jos Metropolis, Nigeria," African Journal of Environmental Science and Technology, vol. 3, no. 12, 2009.

[45] E. Niyongabo, Y.-C. Jang, D. Kang, and K. Sung, "Current treatment and disposal practices for medical wastes in Bujumbura, Burundi," Environmental Engineering Research, vol. 24, no. 2, pp. 211-219, 2018.

[46] S. Sarkar, M. A. Haque, and T. A. Khan, "Hospital waste management in Sylhet city," ARPN Journal of Engineering and Applied Sciences, vol. 1, no. 2, pp. 32-40, 2006. 